\title{
Characterising the proximal patellar tendon attachment and its relationship to skeletal maturity in adolescent ballet dancers
}

\author{
Aliza Rudavsky ${ }^{1}$ \\ Jillianne Cook ${ }^{3}$ \\ Stig Peter Magnusson ${ }^{1,2}$ \\ Michael Kjaer ${ }^{1}$ \\ Sean Docking ${ }^{3}$ \\ 1 Institute of Sports Medicine Copenhagen, \\ Department of Orthopedic Surgery M, Bispebjerg \\ Hospital and Center for Healthy Aging, Faculty of \\ Health and Medical Sciences, University of \\ Copenhagen, Denmark \\ 2 Musculoskeletal Rehabilitation Research Unit, \\ Department of Physical Therapy, Bispebjerg Hospital, \\ Copenhagen, Denmark \\ ${ }^{3}$ La Trobe Sport and Exercise Medicine Research \\ Centre, La Trobe University, Melbourne,Victoria, \\ Australia
}

Corresponding author:

Aliza Rudavsky

Clinical Medicine,

University of Copenhagen

2400 Copenhagen, Denmark

E-mail: aliza.rudavsky@gmail.com

\section{Summary}

Background: It is unknown how and when the proximal attachment of the patellar tendon matures; puberty may be key in ensuring normal tendon formation. The aim of this study was to investigate the features of the proximal patellar tendon attachment at different stages of skeletal maturity, to help gain an understanding of how and when the tendon attachment matures.

Methods: Sixty adolescent elite ballet students (ages 11-18) and eight mature adults participated. Peak height velocity (PHV) estimated skeletal maturity. Ultrasound tissue characterisation (UTC) scan was taken of the left knee and analysed for stability of echopattern. An image-based grading scale for greyscale ultrasound was developed to describe the tendon appearance. Anterior-posterior thickness was measured at the inferior pole of the patella, 1 and 2 centimetres distally. Outcomes were compared with skeletal maturity.

Results: Mid-portion patellar tendon thickness increased with skeletal maturity $(p=0.001$ at $1 \mathrm{~cm}$ and $\mathrm{p}=0.007$ at $2 \mathrm{~cm}$ ). There was more variance in structural appearance (greyscale classification and UTC echopattern) in pre and peri-PHV participants. Tendon attachment one-year post PHV appeared similar to mature tendons.

Conclusions: Early adolescence was associated with highly variable tendon appearance, whereas the tendon appeared mature after PHV. Adolescence may be a critical time for the formation of normal tendon attachment.

Level of evidence: Ilb individual cohort study.

KEY WORDS: ballet students, jumper's knee, jumping athletes, patellar tendon development, skeletal maturity, ultrasound tissue characterisation.

\section{Introduction}

Patellar tendinopathy, also known as "jumper's knee", is the clinical condition of pain and dysfunction, predominantly at the attachment of the patellar tendon to the patella that is associated with jumping and sports with explosive movements. The relationship between jumper's knee symptoms and tendon pathology seen on imaging is complex; of those who present clinically with pain, $79 \%$ have patellar tendon pathology on ultrasound imaging (fusiform swelling and/or a hypoechoic region) ${ }^{1}$. The presence of pathology on imaging is one of several risk factors for pain and dysfunction, yet there is no direct relationship between pathology and the development of symptoms ${ }^{2}$.

While changes on imaging are considered a risk factor for developing symptoms, the aetiology of tendon pathology is not known. Pathological changes of the patellar tendon on imaging are seen in adults, but it is unknown if the pathology developed close to the onset of symptoms or if the pathology developed during adolescence. Interestingly, the proportion of adolescent basketball players (aged 14-18 years old) with pathological patellar tendons is similar to that in the adult population (26\% in adolescents compared to $\sim 30 \%$ in adults) ${ }^{1}$. Furthermore, athletes without pathological changes by the age of 16 have a low risk of developing jumper's knee in adulthood ${ }^{3}$. These data suggest that adolescence may be critical in the development of patellar tendon pathology.

Most of what is understood about patellar tendon maturation is based on general tendon development. Tendons elongate and thicken during postnatal development at a rate proportional to the growth of their 
associated muscles and bones ${ }^{4-6}$. By the time adolescents reach approximately 17 years old, the collagen in the tendon matrix is relatively stable ${ }^{7}$, which is typically after a person has passed their peak height velocity ${ }^{8}$. Several studies have investigated how the patellar tendon matures; however knowledge is currently limited to the tibial attachment ${ }^{9-13}$ with very few investigations at the patella attachment. The appearance of the proximal patellar tendon attachment throughout skeletal maturity and growth is currently unknown.

The aim of this study was to compare the varied appearances of the proximal patellar tendon attachment in young jumping athletes (ballet dancers) across different stages of skeletal development, which was estimated by peak height velocity. The proximal patellar tendon attachment was assessed using Ultrasound Tissue Characterisation (UTC), which allowed for the grey-scale ultrasound appearance to be described visually as well as quantified by the echopattern. As jumper's knee affects primarily jumping athletes, due to high energy storage within the tendon, this study only looked at ballet dancers. This is a preliminary study to determine whether the appearance and UTC echopattern of the proximal patellar tendon attachment differs between various stages of skeletal development. Our hypothesis was that the proximal patellar tendon attachment would transition to a mature appearance during peak height velocity.

\section{Materials and methods}

Ballet students from the Australian Ballet School and Victorian College of the Arts Secondary School between the ages of 11 and 18 participated in this study. All participants were regularly involved in daily dance classes. Anthropometric measurements including height $(\mathrm{cm})$, weight $(\mathrm{kg})$ and sitting height $(\mathrm{cm})$ were collected. For sitting height, participants were sitting tall on a table, thighs fully supported on the table and feet supported so knees were comfortably flexed to $90^{\circ}$. The measurement of sitting height was taken from the ischial tuberosity to the top of the participant's head. Leg length was calculated by subtracting sitting height from standing height. Using leg length, height, weight and age, maturity offset was calculated to estimate peak height velocity based on the equation by Mirwald et al. ${ }^{8}$.

Eight healthy participants between 21-40 years old who had their patellar tendons imaged previously for other studies were also included. No anthropometric data were available for this group, however because of their ages, they will be assumed to be skeletally mature as the average age to reach skeletal maturity in girls is 14.9 and 15.4 in boys ${ }^{14}$.

Participants were categorised into three groups based on the number of years before or after peak height velocity (maturity offset). Participants who were greater than or equal to a year before reaching $\mathrm{PHV}$ estimate were categorised as pre-PHV, between one year before and one year after PHV estimate were considered peri-PHV and those greater than or equal to one year after PHV estimate were labeled post-PHV. The older group (21-40) was considered skeletally mature. The Monash University ethics committee approved this study and all participants, or participants' parents if aged under 16 years, gave informed consent. This study meets the ethical standards of the Journal ${ }^{15}$.

\section{Ultrasound Tissue Characterisation}

UTC is a validated measure with a high degree of inter and intra-rater reproducibility ${ }^{16}$. The UTC scans were taken using a standardised protocol described below: this protocol was adapted from the protocol used for the Achilles tendon 16, 17. The UTC device has a standardised transducer tilt angle that provides a 3-dimensional image of the entire tendon. The image is analysed based on a computerised algorithm that has also been validated against equine tendon tissue samples ${ }^{18-20}$

One investigator (AR) performed all UTC scans using the standardised protocol on each participants' left knee. Participants were in a supine position with the left knee flexed to $90^{\circ}$ in order to position the UTC tracker perpendicular to the patellar tendon. The decision to analyse only the left leg was an a priori decision. While ballet dancers have dominant leg preferences most exercises and movements are performed equally on the right and left legs. For the mature participants, the patellar tendon without pathology and pain was selected and scanned and analysed using the same procedure. A linear-array ultrasound transducer (SmartProbe 10L5, Terason 2000+; Teratech) mounted in a tracking device with a motor-drive and built-in acoustic-coupling stand-off pad (UTC Tracker, UTC Imaging) was placed directly on the patellar tendon such that the ultrasound head was perpendicular to the tendon at the patellar insertion. Once a clear image of the tendon and the inferior pole was apparent and the alignment of the standoff pad was verified visually, the transducer was automatically moved distally over the length of the tendon capturing a transverse greyscale image every $0.2 \mathrm{~mm}$ over $12 \mathrm{~cm}$. This procedure is repeated in the same standardised manner for all participants.

UTC captures 600 contiguous transverse ultrasound images and renders a three-dimensional greyscale image $^{20}$. Based on the captured images, dedicated software analyses the stability of the echopattern across multiple transverse images categorising the tendon into four echo-types (UTC2010, UTC Imaging), with echo-type I being the most stable and echotype IV being the least stable ${ }^{20}$. Echo-type I, II, III and IV are represented as green, blue, red and black pixels respectively, where echo-type I and II are considered aligned fibrilar structure and echo-type III and IV represent disorganised structure ${ }^{16,20,21}$. Histopathological specimens from the horse have been correlated with UTC echopatterns to show it is a valid modality to estimate pathology20.

The region of interest $(\mathrm{ROI})$ for the patellar tendon was selected from the disappearance of the inferior 
pole of the patella, extending two centimetres distally. Manually selected contours were placed along the length of the tendon in intervals no more than $4 \mathrm{~mm}$. The UTC software automatically interpolated contiguous ROls creating a tendon volume where the proportions of each echo-type were calculated. Quantification of the UTC echopattern was performed by the same investigator (AR) blinded to participant identity and age, with the window size set at 25 (slice-thickness $4.8 \mathrm{~mm}$ ). All contours were reviewed by another trained blinded researcher (SD) to ensure consistency.

\section{Subjective maturation grading}

A classification system was developed to categorise the varied appearances of tendon attachments on greyscale imaging. The categories were based on greyscale imaging features in the sagittal (Fig. 1), transverse (Fig. 2) and coronal (Fig. 3) planes. The figures below show the criteria for each score and the total was summed for a total score, then assigned to a "greyscale category." Inter-rater reliability testing was done between two researchers (AR and SD) where 30 UTC scans, selected at random, were independently scored by each rater who was blind to all other analysis.

In the sagittal plane, the tendon was graded a 0 if there was a clear discontinuity between the inferior pole of the patella and the tendon, a 1 if there was some hypoechoic, hyperechoic or normoechoic tis- sue, but not continuous between the inferior pole and tendon and a 2 if there was a clear normoechoic continuation of the tendon over the inferior pole of the patella (Fig. 1).

In the transverse plane as the inferior pole disappeared, grade 0 was assigned for the presence of a hypoechoic region with well-defined borders. It is hypothesised that these regions are cartilage and are not pathology due to the lack of red and black echotypes on the rendered UTC image. Grade 1 was for images with some light grey or variable echogenic areas without clear borders and grade 2 was for a normal echogenic tendon with no areas of hypoechogenecity (Fig. 2).

In the coronal plane researchers graded the images based on the presence or absence of a dark halo around the lower half of the patella. If there was a dark and/or thick halo around the inferior half of the patella it was graded 0 , if there was a thin, light grey or partial halo around the inferior half of the patella it was graded 1 and if there was no halo it was graded 2 (Fig. 3).

\section{Statistical analysis}

Participants were excluded if they had pathology in their tendons. Pathology within their tendon was defined as the presence of a hypoechoic area on greyscale US, focal thickening of the tendon and/or thickness greater than $7 \mathrm{~mm}^{22}$. For the greyscale

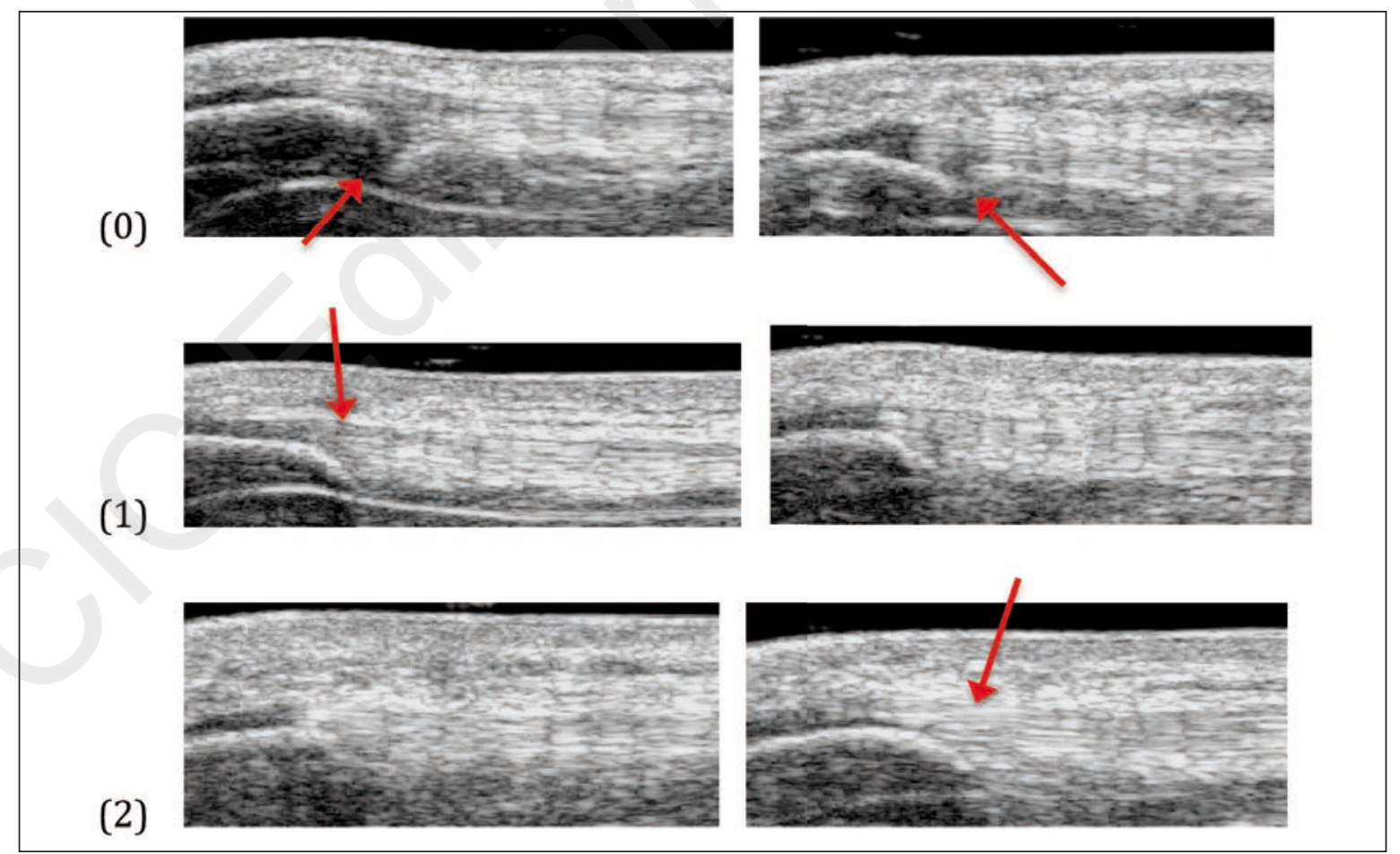

Figure 1. Continuity of tendon fibres at inferior pole of patella (sagittal plane).

(0) Clear discontinuity (between inferior pole of patella and tendon)

(1) Nearly continuous (inconsistent or light grey between inferior pole of the patella and tendon)

(2) Clearly continuous (consistent between patella and tendon) 
Characterising the proximal patellar tendon attachment and its relationship to skeletal maturity in adolescent ballet dancers

(0)
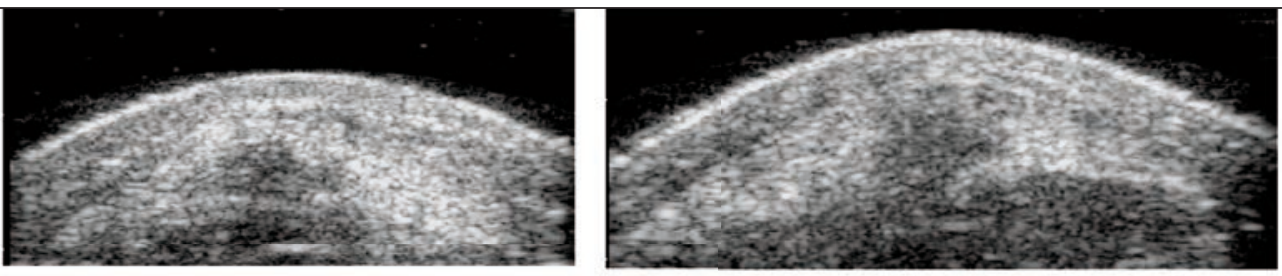

(1)
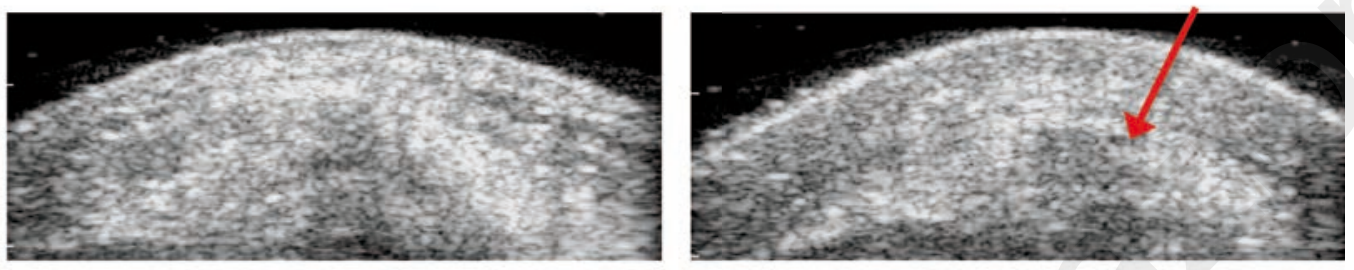

(2)
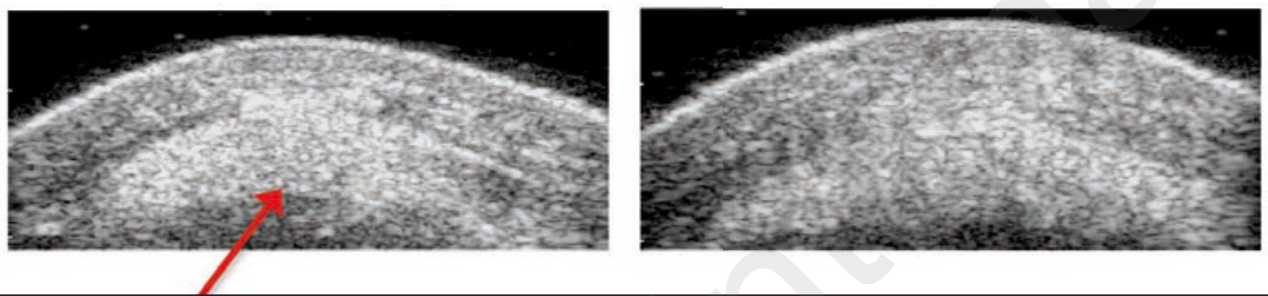

Figure 2. Presence of hypoechoic area immediately distal to inferior pole (transverse plane).

(0) Large, dark grey hypoechoic area.

(1) Light grey hypoechoic/variable echogenic area.

(2) No hypoechoic area.

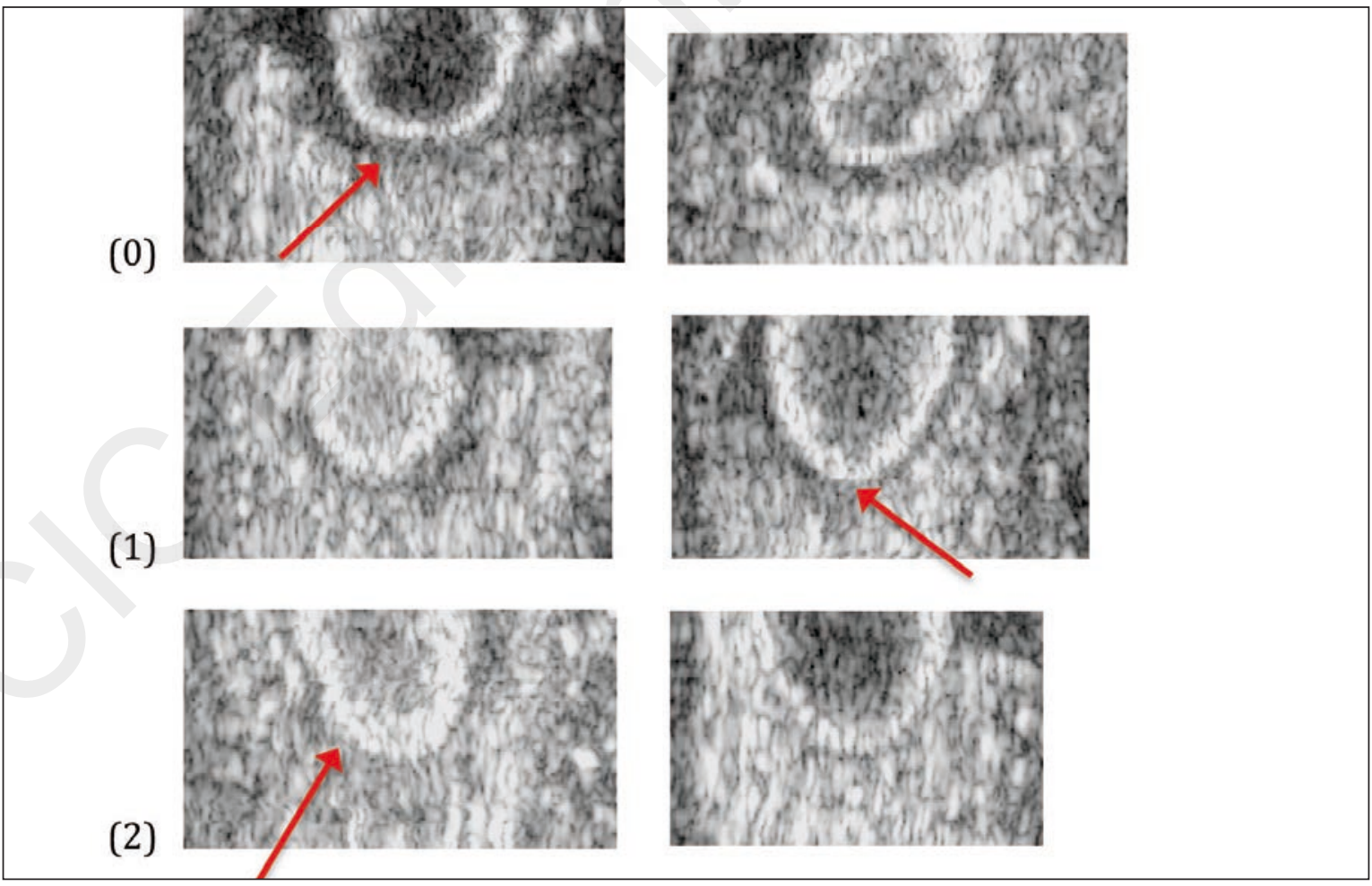

Figure 3. Presence of halo around the inferior half of the patella (coronal plane).

0 . Dark grey halo (around inferior half of patella).

1. Light grey/thin halo (may be thin or partial halo on center or sides).

2. No halo present. 
classification, the totals of each of the three categories were summed (named the patellar attachment score) and placed into three groups: patellar attachment score of 0-2 was classified as category 1 , score of 3-4 was classified as category 2 and score of 5-6 was labeled category 3 . Chi-square testing analysed the arbitrary groups with the maturity offset groups. Levene's test for homogeneity of variance and oneway ANOVA was used to compare maturity offset with the UTC echopattern for the contoured region of interest. One-way ANOVA was also used to compare maturity offset with anterior-posterior thickness across the patella, at the inferior pole, $1 \mathrm{~cm}$ and $2 \mathrm{~cm}$ distal from the inferior pole.

\section{Results}

Of the 60 participants (35 women and 25 men), 23 were pre-PHV, 28 peri-PHV, 9 post-PHV and 8 were mature (Tab. I). After UTC scans were reviewed for quality, where 3 scans were excluded due to poor imaging quality, 65 tendon scans were included; 57 from the ballet students, 8 from the mature group. No pathology was observed in any of the scans.

\section{Anterior-posterior (AP) thickness}

The AP thickness increased by group as PHV increased at $1 \mathrm{~cm}$ and $2 \mathrm{~cm}$ from the inferior pole of the patella ( $p=.001$ and $p=.007$ respectively). However, the AP thickness at the inferior pole of the patella did not differ significantly between the maturity groups $(p=.132)($ Tab. I).

\section{Greyscale scoring and grouping}

Inter-rater reliability demonstrated good agreement with a weighted kappa score of 0.617 . The arbitrary grouping increased with skeletal maturity $(p=.024$, Tab. II). Within the lower maturity offset groups (pre and peri-PHV) there was a distribution of greyscale scores whereas in the higher maturity offset category (post-PHV) and the mature group most participants were in greyscale category three.

\section{UTC echopattern}

There were no significant differences in echo-type across the maturity groups (Tab. III). However, in the pre-PHV and peri-PHV groups there was a greater amount of variability in the echopattern, the variance reduced significantly $(p=0.028)$ in the post and mature groups for the percentage of echo-type I (Fig. 4). Further, there was a trend towards increasing percentage of echo-type I and decreasing percentage of echo-type II seen across skeletal maturity (Tab. III).

\section{Discussion}

This study investigated the varied appearances of the proximal patellar tendon attachment during adolescence to gain further understanding of how and when the proximal patellar tendon matures. We hypothe- sised that the tendon would transition to a mature attachment during this developmental stage. This study identified four main findings: 1) the tendon does not change significantly in thickness directly at the inferior pole, yet increases in thickness with maturity closer to the midsubstance of the tendon; 2) there is a higher degree of variance in the structural composition, as quantified by UTC, at the proximal patellar tendon attachment in the pre and peri-PHV participants; 3) the tendon attachment has a similar appearance in the post PHV and mature group; 4) the greyscale classification system corresponded to the latter stages of maturity offset (post-PHV and mature) with the earlier stages appearing more variable.

This study showed that the appearance of the tendon attachment differed before and after peak height velocity on greyscale imaging. The variable and more hypoechoic appearance in pre- and peri-PHV participants became more normechoic and continuous across the patella in the sagittal and transverse plane in post-PHV and mature groups. While we are limited by these cross-sectional data, the progression towards normal echogenic imaging on US appeared to occur in an anterior to posterior direction with maturity. The posterior aspect may be the last region to mature, which may make it vulnerable to mechanical loading in the pre and peri-PHV years. Interestingly, hypoechoic areas on ultrasound imaging are frequently observed on the posterior aspect at the patellar insertion in adults with patellar tendinopathy ${ }^{23-25}$. One possible explanation is that the hypoechogenicity is a normal part of tendon attachment development that will disappear with normal skeletal maturation, or if exposed to excessive tensile and/or compressive loads before it has formed a mature attachment, it could develop a pathological attachment.

This progression of normal development has been observed at the tibial end of the patellar tendon; a hypoechoic area on ultrasound is common around peak height velocity and less common post-PHV, demonstrating normal tendon tissue maturation ${ }^{11}$. Similar to the findings at the distal patellar tendon attachment ${ }^{11}$, the implication for the imaging finding may be that not all hypoechoic areas are pathological in pre and peri $\mathrm{PHV}$ athletes but rather these areas may be a sign of skeletal immaturity that will "normalise" following PHV. During adolescence the patellar tendon's tibial attachment is vulnerable to the development of pain which, at this site, is termed Osgood-Schlatter disease (OSD). Using grey-scale ultrasound imaging, Ducher et al. (2010) demonstrated that the tibial end of the patellar tendon matures during puberty. The tibial attachment is a cartilage attachment $\sim 1.8$ years before PHV, enthesial fibrocartilage immediately after $\mathrm{PHV}$, where a mature attachment is seen $\sim 2$ years after $\mathrm{PHV}^{10}$, 11. Interestingly, the development of pain appears to be related to certain stages of the maturation process of the tibial insertion, suggesting that aberrant loading during maturation can have a negative effect on the tendon attachment ${ }^{26}$.

The presence of tendon pathology on imaging represents some risk factor for developing patellar tendon 
Characterising the proximal patellar tendon attachment and its relationship to skeletal maturity in adolescent ballet dancers

Table I. Mean anterior-posterior patellar tendon thickness (in centimeters) by maturity offset category.

\begin{tabular}{lccc}
\hline Maturity Offset Categor & Inferior Pole & $\mathbf{1 ~ c m ~ D i s t a l ~ * ~}$ & 2 cm Distal ** \\
\hline Pre & .44 & .44 & .43 \\
Peri & .45 & .44 & .44 \\
Post & .48 & .47 & .45 \\
Mature & .47 & .54 & .51 \\
\hline
\end{tabular}

Significantly increasing thickness with increasing maturity offset category:

${ }^{*} \mathrm{P}=0.001$ at $1 \mathrm{~cm}$

${ }^{* *} \mathrm{P}=0.007$ at $2 \mathrm{~cm}$

$(\mathrm{P}=0.132$ at the inferior pole $)$

Table II. Greyscale score group incidence by maturity offset category.

\begin{tabular}{llll}
\hline $\begin{array}{l}\text { Maturity Offset } \\
\text { category }(\mathrm{n})\end{array}$ & $\begin{array}{l}\text { Greyscale } \\
\text { category } \mathbf{1}\end{array}$ & $\begin{array}{l}\text { Greyscale } \\
\text { category 2 }\end{array}$ & $\begin{array}{l}\text { Greyscale } \\
\text { category 3 }\end{array}$ \\
\hline Pre $(21)$ & $3(14 \%)$ & $11(52 \%)$ & $7(33 \%)$ \\
Peri $(27)$ & $0(0 \%)$ & $17(63 \%)$ & $10(37 \%)$ \\
Post $(9)$ & $1(11 \%)$ & $2(22 \%)$ & $6(67 \%)$ \\
Mature $(8)$ & $0(0 \%)$ & $1(12.5 \%)$ & $7(87.5 \%)$ \\
\hline
\end{tabular}

Table III. One-way ANOVA of mean echo-type pattern (with standard deviation included, SD) at various distances from inferior pole of patella by maturity offset groups. Echo-type I being the most aligned, echo-type IV the least aligned.

\begin{tabular}{|c|c|c|c|c|c|c|}
\hline $\begin{array}{l}\text { Distance } \\
\text { from inferior } \\
\text { pole }(\mathrm{cm}) \text { : }\end{array}$ & Echo-type & $\begin{array}{l}\text { Mean } \\
\text { quantity of } \\
\text { echotype } \\
\text { Pre-PHV (SD) }\end{array}$ & $\begin{array}{l}\text { Mean } \\
\text { quantity of } \\
\text { echotype } \\
\text { Per-PHV (SD) }\end{array}$ & $\begin{array}{l}\text { Mean } \\
\text { quantity of } \\
\text { echotype } \\
\text { Post-PHV } \\
\text { (SD) }\end{array}$ & $\begin{array}{l}\text { Mean } \\
\text { quantity of } \\
\text { echotype } \\
\text { Mature (SD) }\end{array}$ & $\begin{array}{l}\text { P-Value } \\
(\mathrm{Cl}=95 \%)\end{array}$ \\
\hline $0-1$ & 1 & $.56(.12)$ & $.54(.12)$ & $.63(.09)$ & $.59(.05)$ & .210 \\
\hline $0-1$ & II & $.42(.11)$ & $.45(.11)$ & $.36(.08)$ & $.40(.06)$ & .187 \\
\hline $0-1$ & III & $.01(.01)$ & $.01(.01)$ & $.01(.01)$ & $.01(.01)$ & .752 \\
\hline $0-1$ & IV & $.00(.01)$ & $.00(.00)$ & $.00(.00)$ & $.00(.00)$ & .504 \\
\hline $1-2$ & I & $.68(.13)$ & $.68(.09)$ & $.77(.07)$ & $.70(.04)$ & .130 \\
\hline $1-2$ & II & $.32(.13)$ & $.31(.09)$ & $.23(.07)$ & $.29(.05)$ & .135 \\
\hline $1-2$ & III & $.00(.01)$ & $.00(.00)$ & $.00(.00)$ & $.01(.00)$ & .214 \\
\hline $1-2$ & IV & $.00(.01)$ & $.00(.00)$ & $.00(.00)$ & $.00(.00)$ & .574 \\
\hline $0-2$ & I & $.62(.11)$ & $.61(.10)$ & $.70(.07)$ & $.65(.04)$ & .127 \\
\hline $0-2$ & II & $.37(.12)$ & $.38(.10)$ & $.30(.07)$ & $.34(.05)$ & .119 \\
\hline $0-2$ & III & $.01(.01)$ & $.01(.01)$ & $.00(.00)$ & $.01(.00)$ & .714 \\
\hline $0-2$ & IV & $.00(.01)$ & $.00(.00)$ & $.00(.00)$ & $.00(.00)$ & .495 \\
\hline
\end{tabular}

pain ${ }^{1,27}$. However, there are a multitude of factors that also contribute to onset of symptoms, where mechanical overload has been shown to be an important risk factor ${ }^{28-34}$. The patellar tendon is an energy-stor- age tendon that is loaded by jumping; hence why symptoms are called "jumper's knee." High volumes of jumping and energy-storage may induce pathology or trigger symptoms in a tendon that is pathological. 


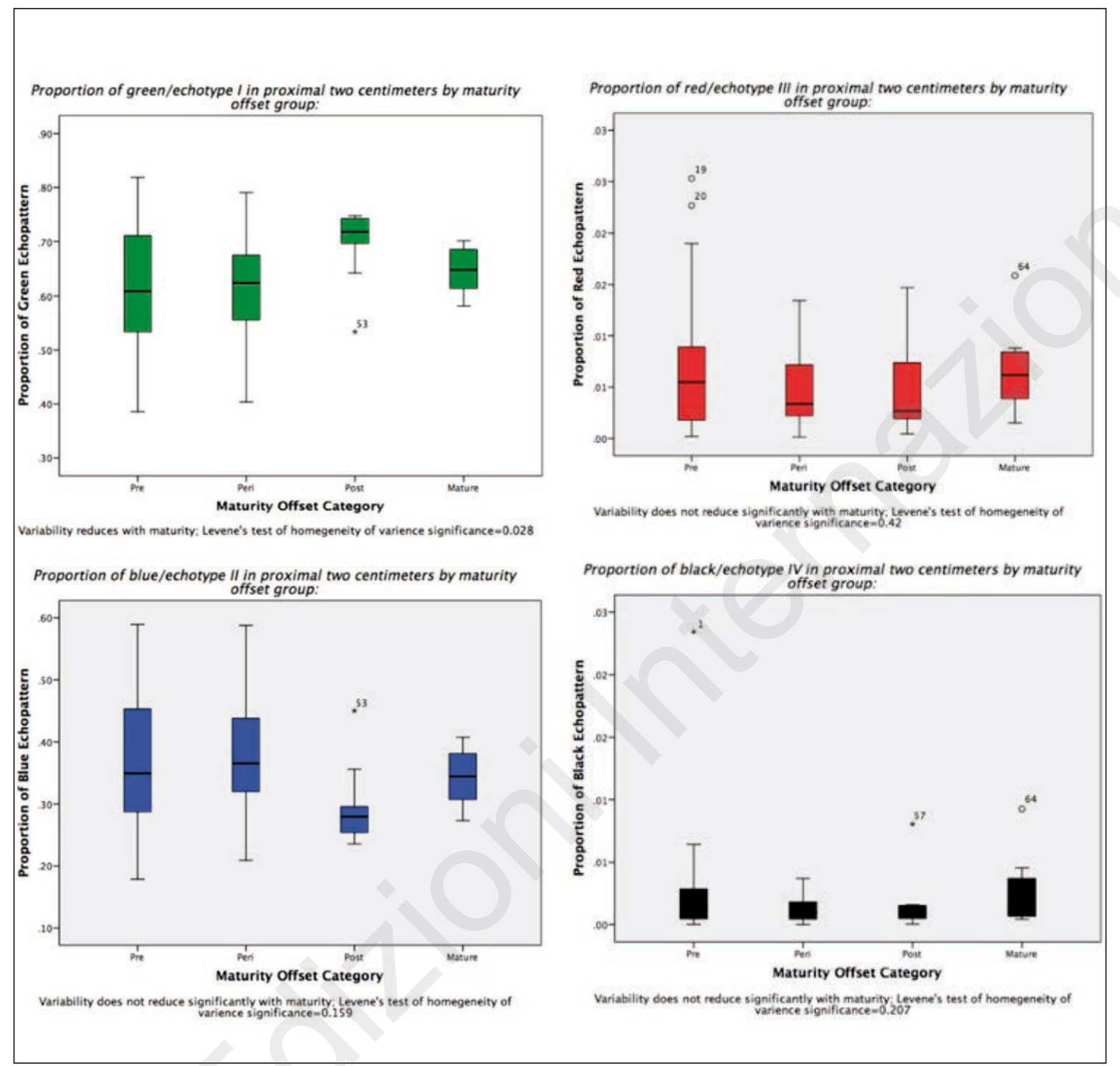

Figure 4. Variance of echopatterns.

Interestingly, dancers complete approximately 200 jumps in each ballet class that may induce pathological changes ${ }^{35}$. While energy-storage is the primary load placed on the patellar tendon, the patellar insertion may also have compressive loads that may be implicated in the development of pathology ${ }^{36}$. Hamilton and Purdam (2004) described hypoechoic areas on the posterior aspect of the attachment as an adaptive response to compressive loads and a potential variation in how the tendon inserts into the patella ${ }^{23}$. This study also reported that the tendon is thicker at 1 and $2 \mathrm{~cm}$ distal to the inferior pole of the patella in mature participants. This is a similar result to the finding of Kubo et al. (2014) who observed in a crosssectional study that the cross-sectional area and length of the patellar tendon increased with larger body size during growth ${ }^{12}$. It is likely that tendon thickness is an adaptation to increasing body mass and muscle strength during puberty.

The variability in the echopattern decreased through maturity at the patellar insertion, particularly echotypes I and II. Based on our current understanding of tendon maturation, it is possible that hyaline or fibrocartilage with type II collagen and larger proteoglycans are present in younger people, which then transitions to mature tendon tissue ${ }^{37}$.

\section{Clinical implications}

Based on these preliminary findings, we see that while there is a high degree of variation in the appearance of the proximal patellar tendon attachment in pre and peri PHV groups, the post PHV tendons appear mature. This supports our hypothesis that there is a transition towards mature tendon attachment during PHV, which is important because once clinicians better understand normal development; 
they can begin to understand abnormal or pathological development, which can lead to pain and dysfunction. This study also provides a method to describe the variations in tendon appearance using the greyscale score with the option to describe all three planes within the tendon. Prospective studies are needed to validate whether the proximal patellar tendon attachment develops during puberty as is proposed and suggested by the current study.

Limitations of this study include only one blind assessor and one blind reviewer evaluating the UTC scans where other studies have used three blind assessors, though reliability of the UTC has been established in previous studies ${ }^{17}$. The greyscale score needs to be validated in a prospective study. Also, there is an unequal distribution of participants between the three peak height velocity groups and the mature groups.

\section{Conclusions}

Based on this cross-sectional study, we found that leading up to peak height velocity the tendon has a variable appearance compared to post peak height velocity. While tendon development is not solely dependent on peak height velocity and other factors may influence maturation, it does appear that pre and peri PHV are important stages for the transition of the proximal patellar tendon attachment to mature tendon. Prospective research is needed to improve our understanding of how the proximal patellar tendon attachment develops throughout skeletal maturity, and whether the stage of tendon development impacts the risk of developing tendon pathology as has been shown at the distal attachment ${ }^{10,26}$.

\section{Acknowledgements}

This research received no specific grant from any funding agency in the public, commercial, or not-forprofit sectors.

\section{References}

1. Cook JL, Khan KM, Kiss ZS, Griffiths L. Patellar tendinopathy in junior basketball players: a controlled clinical and ultrasonographic study of 268 patellar tendons in players aged 14-18 years. Scandinavian journal of medicine \& science in sports. 2000;10(4):216-20.

2. Cook JL, Khan KM, Harcourt PR, Kiss ZS, Fehrmann MW, Griffiths $\mathrm{L}$, et al. Patellar tendon ultrasonography in asymptomatic active athletes reveals hypoechoic regions: a study of 320 tendons. Victorian Institute of Sport Tendon Study Group. Clinical journal of sport medicine: official journal of the Canadian Academy of Sport Medicine. 1998;8(2):73-7.

3. Gisslen K, Gyulai C, Nordstrom P, Alfredson H. Normal clinical and ultrasound findings indicate a low risk to sustain jumper's knee patellar tendinopathy: a longitudinal study on Swedish elite junior volleyball players. British journal of sports medicine. 2007;41(4):253-8.

4. Crawford GN. An experimental study of tendon growth in the rabbit. The Journal of bone and joint surgery British volume. 1950;32-B(2):234-43.

5. Elliott DH. Structure and Function of Mammalian Tendon. Biological reviews of the Cambridge Philosophical Society. 1965;40:392-421.

6. Hughes $\mathrm{H}$. An experimental study of the post-natal growth of tendon. Anatomischer Anzeiger. 1956;103(10-12):192-7.

7. Heinemeier KM, Schjerling P, Heinemeier J, Magnusson SP, Kjaer M. Lack of tissue renewal in human adult Achilles tendon is revealed by nuclear bomb (14)C. FASEB journal: official publication of the Federation of American Societies for Experimental Biology. 2013;27(5):2074-9.

8. Mirwald RL, Baxter-Jones AD, Bailey DA, Beunen GP. An assessment of maturity from anthropometric measurements. Medicine and science in sports and exercise. 2002;34(4):68994.

9. Cassel M, Baur H, Hirschmuller A, Carlsohn A, Frohlich K, Mayer F. Prevalence of Achilles and patellar tendinopathy and their association to intratendinous changes in adolescent athletes. Scandinavian journal of medicine \& science in sports. 2014.

10. Ducher G, Cook J, Lammers G, Coombs P, Ptazsnik R, Black $\mathrm{J}$, et al. The ultrasound appearance of the patellar tendon attachment to the tibia in young athletes is conditional on gender and pubertal stage. Journal of science and medicine in sport / Sports Medicine Australia. 2010;13(1):20-3.

11. Ducher G, Cook J, Spurrier D, Coombs P, Ptasznik R, Black J, et al. Ultrasound imaging of the patellar tendon attachment to the tibia during puberty: a 12-month follow-up in tennis players. Scandinavian journal of medicine \& science in sports. 2010;20(1):e35-40.

12. Kubo K, Teshima T, Hirose N, Tsunoda N. Growth changes in morphological and mechanical properties of human patellar tendon in vivo. Journal of applied biomechanics. 2014;30 (3):415-22.

13. Pearson SJ, Hussain SR. Region-specific tendon properties and patellar tendinopathy: a wider understanding. Sports medicine. 2014;44(8):1101-12.

14. Howell FR, Mahood JK, Dickson RA. Growth beyond skeletal maturity. Spine. 1992;17(4):437-40.

15. Padulo J, Oliva F, Frizziero A, Maffulli N. Muscle, Ligaments and Tendons Journal. Basic principles and recommendations in clinical and field science research. MLTJ. 2016;6(1):1-5.

16. Van Schie HT, de Vos RJ, de Jonge S, Bakker EM, Heijboer MP, Verhaar JA, et al. Ultrasonographic tissue characterisation of human Achilles tendons: quantification of tendon structure through a novel non-invasive approach. British journal of sports medicine. 2010;44(16):1153-9.

17. Van Ark M, Docking SI, van den Akker-Scheek I, Rudavsky A, Rio E, Zwerver J, et al. Does the adolescent patellar tendon respond to 5 days of cumulative load during a volleyball tournament? Scandinavian journal of medicine \& science in sports. 2015.

18. Van Schie HT, Bakker EM, Jonker AM, van Weeren PR. Ultrasonographic tissue characterization of equine superficial digital flexor tendons by means of gray level statistics. American journal of veterinary research. 2000;61(2):210-9.

19. Van Schie HT, Bakker EM, Jonker AM, van Weeren PR. Efficacy of computerized discrimination between structure-related and non-structure-related echoes in ultrasonographic images for the quantitative evaluation of the structural integrity of superficial digital flexor tendons in horses. American journal of veterinary research. 2001;62(7):1159-66.

20. Van Schie HT, Bakker EM, Jonker AM, van Weeren PR. Computerized ultrasonographic tissue characterization of equine superficial digital flexor tendons by means of stability quantification of echo patterns in contiguous transverse ultrasonographic images. American journal of veterinary research. 
2003;64(3):366-75.

21. Docking SI, Daffy J, van Schie HT, Cook JL. Tendon structure changes after maximal exercise in the Thoroughbred horse: use of ultrasound tissue characterisation to detect in vivo tendon response. Veterinary journal. 2012;194(3):338-42.

22. Schmid MR, Hodler J, Cathrein P, Duewell S, Jacob HA, Romero J. Is impingement the cause of jumper's knee? Dynamic and static magnetic resonance imaging of patellar tendinitis in an open-configuration system. The American journal of sports medicine. 2002;30(3):388-95.

23. Hamilton B, Purdam C. Patellar tendinosis as an adaptive process: a new hypothesis. British journal of sports medicine. 2004;38(6):758-61.

24. Haraldsson BT, Aagaard P, Krogsgaard M, Alkjaer T, Kjaer M, Magnusson SP. Region-specific mechanical properties of the human patella tendon. Journal of applied physiology. 2005;98 (3):1006-12.

25. Scott A, Docking S, Vicenzino B, Alfredson H, Murphy RJ, Carr AJ, et al. Sports and exercise-related tendinopathies: a review of selected topical issues by participants of the second International Scientific Tendinopathy Symposium (ISTS) Vancouver 2012. British journal of sports medicine. 2013;47(9):53644.

26. Sailly M, Whiteley R, Johnson A. Doppler ultrasound and tibial tuberosity maturation status predicts pain in adolescent male athletes with Osgood-Schlatter's disease: a case series with comparison group and clinical interpretation. British journal of sports medicine. 2013;47(2):93-7.

27. Docking SI, Ooi CC, Connell D. Tendinopathy: Is Imaging Telling Us the Entire Story? The Journal of orthopaedic and sports physical therapy. 2015:1-27.

28. Magnusson SP, Langberg $\mathrm{H}$, Kjaer M. The pathogenesis of tendinopathy: balancing the response to loading. Nat Rev Rheumatol. 2010;6(5):262-8.
29. Cook JL, Purdam CR. Is tendon pathology a continuum? A pathology model to explain the clinical presentation of load-induced tendinopathy. British journal of sports medicine. 2009;43(6):409-16.

30. Culvenor AG, Cook JL, Warden SJ, Crossley KM. Infrapatellar fat pad size, but not patellar alignment, is associated with patellar tendinopathy. Scandinavian journal of medicine \& science in sports. 2011;21(6):e405-11.

31. Edwards S, Steele JR, McGhee DE, Beattie S, Purdam C, Cook JL. Landing strategies of athletes with an asymptomatic patellar tendon abnormality. Medicine and science in sports and exercise. 2010;42(11):2072-80.

32. Ferretti A. Epidemiology of jumper's knee. Sports medicine 1986;3(4):289-95.

33. Van der Worp H, van Ark M, Zwerver J, van den Akker-Scheek I. Risk factors for patellar tendinopathy in basketball and volleyball players: a cross-sectional study. Scandinavian journal of medicine \& science in sports. 2012;22(6):783-90.

34. Witvrouw E, Bellemans J, Lysens R, Danneels L, Cambier D. Intrinsic risk factors for the development of patellar tendinitis in an athletic population. A two-year prospective study. The American journal of sports medicine. 2001;29(2):190-5.

35. Liederbach M, Richardson, M., Rodriguez, M., Compagno, J., Dilgen, F. E., \& Rose, D. J. Jump exposures in the dance training environment: a measure of ergonomic demand. Journal of athletic training. 2006;41(2):S85.

36. Cook JL, Purdam C. Is compressive load a factor in the development of tendinopathy? British journal of sports medicine. 2012;46(3):163-8.

37. Benjamin M, Ralphs JR. Functional and developmental anatomy of tendons and ligaments. In: Gordon SL, Blair SJ, Fine LJ, editors. Repetitive Motion Disorders of the Upper Extremity. Rosemont, IL: American Academy of Orthopaedic Surgeons; 1995. 